\title{
Fate of Untreated Adjacent Spondylolysis in Selectively Surgically Treated Patients with Multi-Level Spondylolysis: Should All Segments Always be Fused?
}

\author{
Se-Jun Park, Kyung Hyun Kim, Moo-Sung Kang, Jeong-Yoon Park, Sung-Uk Kuh, Dong-Kyu Chin, \\ Keun-Su Kim, Yong-Eun Cho \\ Department of Neurosurgery, Spine and Spinal Cord Institute, Gangnam Severance Hospital, Yonsei University \\ College of Medicine, Seoul, Republic of Korea
}

Corresponding author: Kyung Hyun Kim Department of Neurosurgery, Spine and Spinal Cord Institute, Gangnam Severance Hospital, Yonsei University College of Medicine, 211, Eonjuro, Gangnam-gu, Seoul 06273, Republic of Korea

Tel: +82-2-2019-3390

Fax: +82-3-3461-9229

E-mail: nskhk@yuhs.ac

Received: June 8, 2020

Revised: July 19, 2020

Accepted: August 20, 2020
Objective: The purpose of this study was to observe the natural course of remained untreated spondylolysis adjacent to previous fusion segments in patients with multi-level lysis and its clinical outcome. Methods: Nineteen patients who underwent selective fusion of multi-level spondylolysis (MLS) at a single institute were enrolled. As a matched cohort for comparison, 19 patients who had single-level spondylolysis (SLS) and undergone singlelevel fusion with similar demographics and preoperative radiologic measurements as the MLS group were included. We evaluated the preoperative, postoperative, and last followup angular displacement and sagittal translation on dynamogram radiographs, and axial and radial pain using the visual analogue scale in both groups. We then compared the increment in radiological instability and clinical outcome between the MLS and SLS groups. Results: There were no significant differences in patient demographics and preoperative radiological measurements, including disc degeneration, facet degeneration, lumbar lordosis, pelvic incidence, and sacral table angle between both groups. Both groups showed an increase in the average angular displacement and slippage during the final follow-up as compared to preoperative findings, but no significant difference was noticed between them. Both the MLS and SLS groups showed improvement in lower back pain and leg pain from before surgery, but with no statistical significance. Conclusion: Selective fusion in patients with multiple spondylolysis can be an alternative surgical option without increasing the risk of adjacent segment degeneration under strict narrow indications. However, a thorough preoperative evaluation is needed to prevent early surgical failure.

Key Words: Spinal fusion; Spondylolysis; Spondylolisthesis

\section{INTRODUCTION}

Spondylolysis is defined as a defect or fracture of the pars interarticularis of the vertebra, commonly affecting the lumbar and lumbosacral vertebrae ${ }^{5,7)}$. The pathogenesis of lysis is still not clearly understood, but many factors, including mechanical, genetic, hormonal factors could have an impact ${ }^{7}$. Lytic lesion makes the related vertebral body vulnerable to slippage, leading to isthmic listhesis ${ }^{7}$. According to previous studies, the overall incidence of lysis is about $3 \%$ to $6 \% 0^{3,24)}, 2.6 \%$ to $6.0 \%$ for isthmic listhesis ${ }^{7,18)}$, and only $0.3 \%$ to $1.48 \%$ for multi-level spondylolysis $(\mathrm{MLS})^{21,22)}$. The incidence of spondylolysis and isthmic listhesis can vary depending on age, sex, ethnicity, and congenital abnormalities ${ }^{7,24)}$.

Beutler et al. ${ }^{3)}$ documented that patients with bilateral pars defect developed listhesis, but showed slow progression with each decade. In addition, no significant correlation was noted between the degree of slippage and clinical symptoms ${ }^{3)}$. Most of the patients are asymptomatic, and even in the symptomatic patients, conservative care is usually adequate in dealing with the symptoms ${ }^{5,7)}$. Due to its relatively benign character, most of the patients with stable lesions do not need surgical treatment. However, patients with intractable pain, neurological deficits despite non-operative treatment with or without progressive slip, and segmental instability are considered potential candidates for surgery $7,17,25)$

Many surgical approaches including direct repair of the pars, and decompression and fusion with or without instrumentation have been introduced for the treatment of spondylolysis and listhesis ${ }^{4,5,16,18)}$. Changes in the adjacent segment after fusion of single-level and isthmic listhesis have been reported by 
many authors ${ }^{2,23,29)}$. It is known that increased stress is applied at the adjacent level of fusion ${ }^{11)}$, and that fusion length can affect the progression of degeneration of the adjacent segment ${ }^{8,26)}$. However, studies on treatment and clinical outcomes of patients with multi-level lysis are extremely rare ${ }^{14,30)}$. Our institute tried to limit the fusion segment in multiple spondylolysis by performing operations selectively at the symptomatic level. By limiting the fusion length, it minimizes the operation time, intraoperative blood loss, and adjacent segment degeneration. The aim of this study was to observe the natural history of untreated spondylolysis adjacent to previous fusion in patients with multi-level lysis and the clinical outcome.

\section{MATERIALS AND METHODS}

\section{Patients}

From January 2004 to April 2014, 762 consecutive patients were surgically treated for a diagnosis of spondylolytic spondylolisthesis at our institute. Of these, $11.0 \%(n=84)$ of the patients showed MLS, with 3 patients presenting triple-level lysis. The L4 and L5 were the most commonly affected levels in double level lysis patients $(n=66)$, while all cases of triple-level lysis affected the L3, L4, and L5 levels (n=3) (Fig. 1).

Only those patients who underwent selective fusion for symptomatic lytic listhesis out of the multiple spondylolysis cases were included in the study. The criteria of no symptoms, no instability in lateral flexion/extension plain radiographs, no definite stenosis on magnetic resonance imaging (MRI), and no provocative symptoms or release of symptoms with diagnostic nerve root block at the concerned level had to be fulfilled for the corresponding lytic segment to be excluded from the operation. We considered segmental angular discrepancy greater than $10^{\circ}$ between the flexion and extension views on the radiograph or translation more than $3 \mathrm{~mm}$ on the lateral dynamogram as instability ${ }^{29)}$. This group was called the MLS selective fusion group (n=19) (Fig. 2).

Following this, we selected patients who only had single-

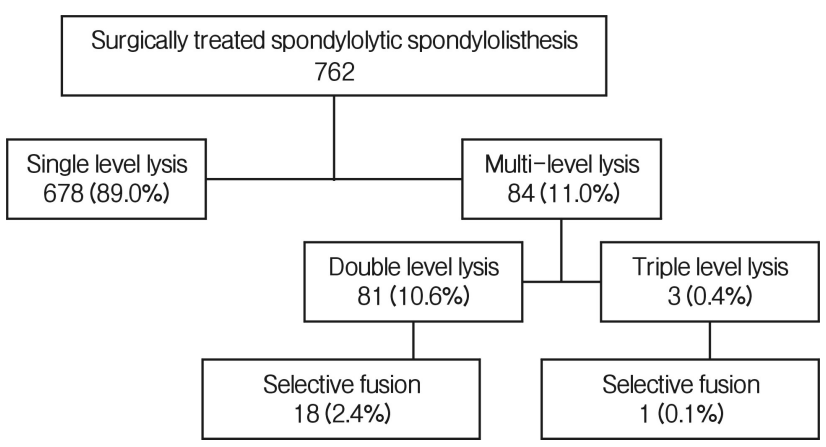

Fig. 1. Patients with multi-level spondylolysis who underwent fusion operation. level lysis and underwent single-level fusion of the corresponding lesion. Patients with similar demographics (age, sex, follow-up period, lysis level) and imaging parameters of the adjacent non-fused segment (disc degeneration, facet degeneration, lumbar lordosis, pelvic incidence, and sacral table angle) were matched with the MLS group patients as a cohort for comparison to reduce the effect caused by confounders. This matched cohort group was called the single-level spondylolysis (SLS) fusion group (n=19) (Fig. 2).

All the patients in the MLS group underwent preoperative, postoperative, and last follow-up lumbar lateral dynamogram plain radiographs, as well as preoperative sagittal and axial computed tomography (CT). Furthermore, all patients, except 2, underwent preoperative sagittal and axial T2 weighted MRI images. Similarly, all patients in the SLS group underwent preoperative, postoperative, and last follow-up lumbar flexion and extension radiographs, as well as preoperative sagittal and axial CT and T2-weighted MRI. A retrospective analysis was conducted on the records of 19 patients in each group to evaluate the axial and radiating pain in the preoperative period and at the last follow-up visit using the visual analogue scale (VAS).

Plain radiographs were used to assess the lumbar lordosis as per the L1-L5 Cobb angle ${ }^{9}$, pelvic incidence by the technique
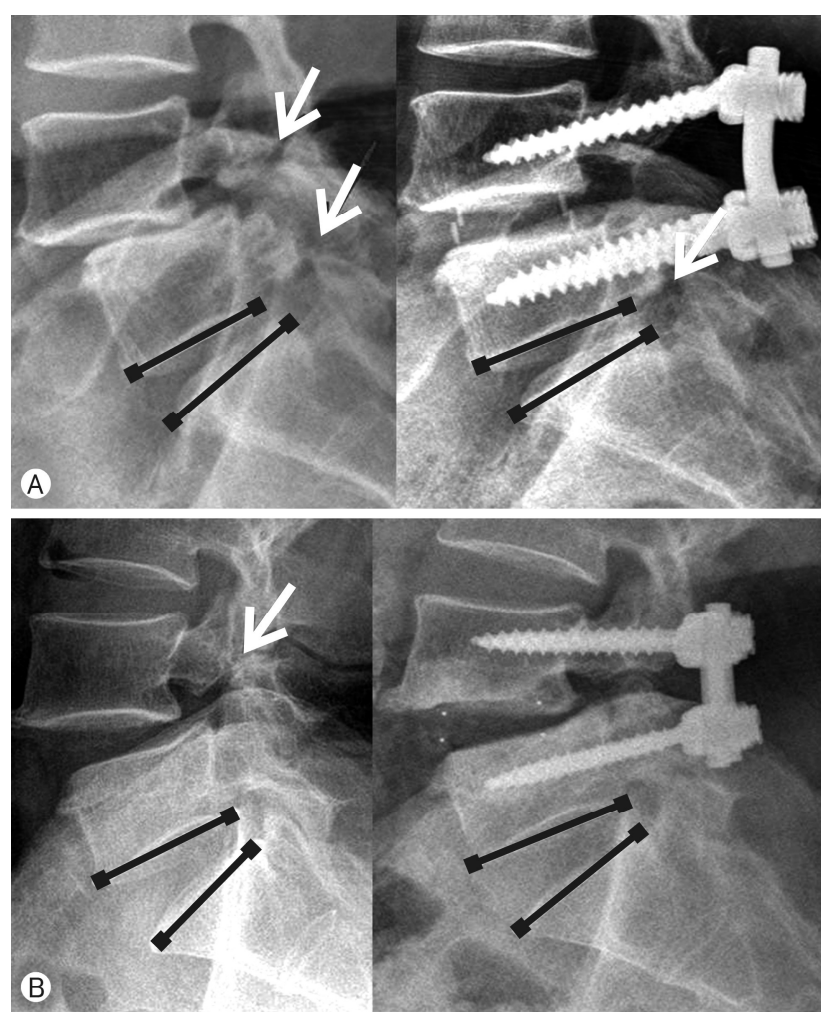

Fig. 2. Pre and postoperative lateral plain radiograph of multi-level spondylolysis (MLS) and single-level spondylolysis (SLS) group. (A) Pre and postoperative lateral plain radiograph in MLS group. (B) Pre and postoperative lateral plain radiograph in SLS group (white arrow: spondylolysis, black line: angular displacement). 
of Legaye et al. ${ }^{13)}$, and sacral table angle as defined by Inoue et al. ${ }^{10)}$ as the angle between the upper endplate with the posterior wall of the lower vertebral body. Disc degeneration was evaluated using Pfirrmann grade ${ }^{19)}$ on the MRI scans, while facet degeneration was evaluated using Weishaupt grade ${ }^{27)}$ on CT images.

The angular displacement, defined as the difference of segmental angle in flexion and extension views, was measured on the lateral dynamogram radiographs between the fused lytic segment and the adjacent untreated lytic segment. The angular displacement and slippage on lateral dynamogram radiographs were measured preoperatively, postoperatively, and at the last follow-up. Increment of angular displacement and translation between postoperative and last follow-up evaluation was calculated for respective groups. The angle between the lower
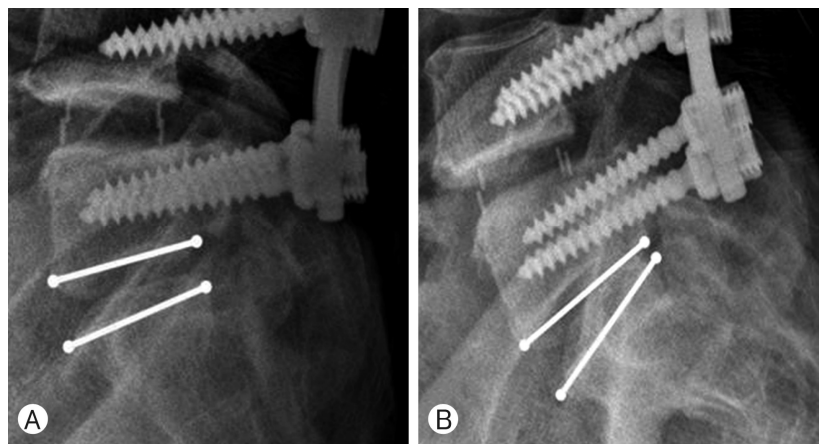

Fig. 3. Measurement of angular displacement on flexion/extension plain radiographs. Example of $L 4 / 5$ spondylolysis patient who underwent $L 4 / 5$ selective fusion only. (A) Angle between the lower endplate of the fused vertebra (L5) and the upper endplate of the adjacent non-fused spondylolysis vertebra (S1) in flexion view. (B) Angle between the lower endplate of the fused vertebra (L5) and the upper endplate of the adjacent non-fused spondylolysis vertebra (S1) in extension view. Angular displacement $\left({ }^{\circ}\right)$ : difference between $(A)$ angle in flexion view and $(B)$ angle in extension view on dynamogram radiograph.
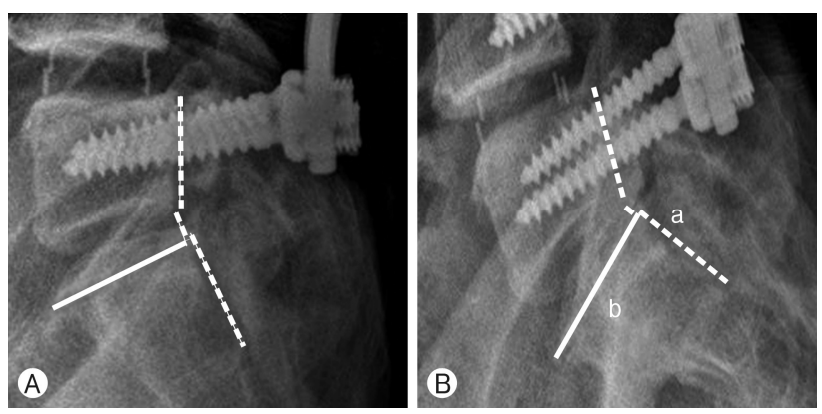

Fig. 4. Calculation of translation on flexion/extension plain radiographs. (A) Length of anterior displacement of the upper vertebra. (B) Length of upper endplate of the lower vertebra (white dotted line: posterior margin of vertebral body). Sagittal plane translation (\%): difference between $a / b \times 100$ in flexion view and $a / b \times 100$ value in extension view on dynamogram radiograph. endplates of the upper vertebra and upper endplates of the lower vertebras was measured by the Posner's method ${ }^{20)}$ (Fig. 3). The sagittal translation on the dynamogram was measured using the Dupuis technique ${ }^{(6)}$ by calculating the ratio of anterior translation of the upper segment in relation to the length of the upper endplate of the lower vertebra (Fig. 4).

\section{Statistical Analysis}

The normality test using the Kolmogorov-Smirnov test proved that increment of angular displacement and slippage was not normally distributed; hence, Mann-Whitney U-test was used for comparison between the MLS and SLS groups. Ordinal variables (disc and facet degeneration) were also compared using the Mann-Whitney U-test. Other variables were confirmed as normally distributed; therefore, an independent $t$-test was used to compare both groups. The Statistical Package for the Social Sciences software (version 20.0; SPSS Inc., Chicago, IL, USA) was used for all statistical analyses. A p-value of less than 0.05 was considered significant.

\section{RESULTS}

\section{Patient Demographics and Radiological Assess- ment of the MLS and SLS Group and Distribution of the Multi-level Lysis Levels}

The MLS group consisted of 5 males and 14 females aged between 32 and 73 years (mean $55.1 \pm 2.4$ years) at the time of the operation. The SLS group consisted of 7 males and 12 females aged between 36 and 72 years (mean $54.9 \pm 2.4$ years). The average time between surgery and the final follow-up

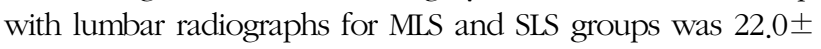
5.6 and 21.8 \pm 3.0 months, respectively. The patient demographics and preoperative imaging measurements of the adjacent non-fused segment did not show a significant difference between the 2 groups. In the MLS group, 7 patients had double-level spondylolysis at L3 and L4 and underwent selective L4/5 fusion, except for one patient who had fusion only at the L3/4 level. Eleven patients with L4 and L5 double-level spondylolysis underwent limited L4/5 fusion, except for one patient with L5/S1 fusion. One patient had triple-level spondylolysis at the L3, L4, and L5 levels and underwent fusion at the L4/5/S1 level. The SLS group had same distribution ratio of the fusion segment matched with the MLS group (Fig. 1, Table 1).

\section{Radiological and Clinical Outcomes in the MLS and SLS Groups}

The mean preoperative angular displacement measured at the adjacent spondylolysis segment in the MLS group was $6.6 \pm$ $5.3^{\circ}$ and increased to $9.0 \pm 5.8^{\circ}$ postoperatively, with a mean 
postoperative increment of $2.4 \pm 1.1^{\circ}$ from post-surgery to the last follow-up. The mean preoperative and postoperative angular displacement values in the SLS group were $7.5 \pm 4.1^{\circ}$ and $10.0 \pm 4.7^{\circ}$, respectively. The postoperative increment from post-surgery to the last follow-up in the SLS group was $1.8 \pm$ $0.9^{\circ}$. The difference in the postoperative increment of angular displacement at the adjacent level between the 2 groups was not statistically significant $(\mathrm{p}=0.097)$ (Table 2).

The mean sagittal plane translation measured at the adjacent spondylolysis segment in the MLS group showed a postoperative increment $1.2 \pm 2.5 \%$, whereas that in the SLS group was $2.5 \pm 4.2 \%$. There was no significant difference in the increase in slippage between both groups from post-surgery to the final follow-up $(\mathrm{p}=0.562)$.

Using the numeric rating scale (NRS) scale, lower back pain showed a decrease from $4.9 \pm 1.3$ preoperatively to $2.7 \pm 1.3$ at the last follow-up in the MLS group, and from $4.3 \pm 1.5$ preoperatively to $1.4 \pm 1.5$ at the last follow-up in the SLS group. Leg pain improved from $6.9 \pm 1.5$ preoperatively to $2.5 \pm 1.3$ at the final follow-up in the MLS group. In the SLS group, the radiating pain reduced from $6.2 \pm 1.4$ preoperatively to $1.4 \pm 1.2$ at the final follow-up. The SLS group showed a greater decrease in axial and radiating pain VAS scores, but there was no statistical difference between the 2 groups.

\section{DISCUSSION}

Multiple level spondylolysis is extremely rare. Sakai et al. ${ }^{22)}$ reviewed the CT scans of 2,000 Japanese people, and reported

Table 1. Patient demographics and radiological assessment in the multi-level spondylolysis selective fusion group and single level spondylolysis fusion group

\begin{tabular}{lccc}
\hline \hline Patients factors & MLS $(\mathrm{n}=19)$ & $\mathrm{SLS}(\mathrm{n}=19)$ & $\mathrm{p}$-value \\
\hline Age & $55.1 \pm 2.4$ & $54.9 \pm 2.4$ & $0.795^{*}$ \\
Sex (Male : Female) & $5: 14$ & $7: 12$ & - \\
Follow-up period & $22.0 \pm 5.6$ & $21.8 \pm 3.0$ & $0.357^{*}$ \\
Disc degeneration (Pfirrmann grade) & & & \\
O & $1(5.3 \%)$ & $0(0.0 \%)$ & $0.749^{+}$ \\
I & $0(0.0 \%)$ & $1(5.3 \%)$ & \\
II & $4(21.1 \%)$ & $6(31.6 \%)$ & \\
III & $5(26.3 \%)$ & $5(26.3 \%)$ & \\
IV & $7(36.8 \%)$ & $7(36.8 \%)$ & \\
Facet degeneration (Weishaupt grade) & & & \\
O & $1(5.3 \%)$ & $5(26.3 \%)$ & $0.162^{+}$ \\
I & $8(42.1 \%)$ & $7(36.8 \%)$ & \\
II & $9(47.4 \%)$ & $7(36.8 \%)$ & \\
III & $1(5.3 \%)$ & $0(0.0 \%)$ & \\
Lumbar lordosis (L1-5) & $37.0 \pm 2.8$ & $33.6 \pm 2.0$ & $0.163^{*}$ \\
Pelvic incidence & $60.0 \pm 2.8$ & $58.1 \pm 1.8$ & $0.256^{*}$ \\
Sacral table angle & $85.8 \pm 0.5$ & $85.4 \pm 0.5$ & $0.624^{*}$ \\
\hline
\end{tabular}

MLS: multi-level spondylolysis; SLS: single-level spondylolysis.

Independent $t$-test. ${ }^{+}$Mann-Whitney U-test.

Table 2. Comparison of radiological measurement and clinical outcomes between the multi-level spondylolysis selective fusion group and single level spondylolysis fusion group

\begin{tabular}{|c|c|c|c|}
\hline Radiologic/Clinical outcomes & $\operatorname{MLS}(n=19)$ & $S L S(n=19)$ & p-value \\
\hline Increment of angular displacement $\left({ }^{\circ}\right)$ & $2.40 \pm 1.10$ & $1.77 \pm 0.93$ & $0.097^{\dagger}$ \\
\hline Increment of sagittal plane translation (\%) & $1.17 \pm 2.50$ & $2.53 \pm 4.16$ & $0.562^{+}$ \\
\hline Change of axial pain (VAS) & $-2.2 \pm 1.9$ & $-2.9 \pm 2.1$ & $0.267^{*}$ \\
\hline Change of radiating pain (VAS) ${ }^{\ddagger}$ & $-4.4 \pm 1.7$ & $-4.7 \pm 1.8$ & $0.578^{*}$ \\
\hline
\end{tabular}

MLS: multi-level spondylolysis; SLS: single-level spondylolysis; VAS: visual analogue scale.

Independent t-test. ${ }^{+}$Mann-Whitney U-test. ${ }^{\ddagger}$ Negative value means decrease of VAS score. 
that $5.9 \%$ had lumbar spondylolysis, and $0.3 \%(\mathrm{n}=5)$ had multilevel pars defect. Ravichandran ${ }^{21)}$ reported that $1.48 \%$ of patients complaining of back pain had multiple lysis. In our study, out of 762 surgically treated isthmic listhesis patients, 84 patients (11\%) had multiple spondylolysis. We believe that since our research was not based on the general population, and the asymptomatic patients and non-surgically treated MLS patients were excluded, it showed higher percentage of multiple lysis patients than the previous reports. It is controversial whether multiple spondylolysis is more common in males or females ${ }^{14,30)}$. In our study, there were more females $(73.7 \%, 14 / 19)$ than males. Preliminary studies have stated that multiple lumbar lysis occurred mostly at L3-L5 levels ${ }^{14,30)}$, which corroborates with our study.

The pars defect tends to progress through childhood and adolescence, but slows down later ${ }^{3,24)}$. Slip progression does not show meaningful correlation with progression of symptoms; therefore, as long as there are no symptoms, the treatment of spondylolysis is unnecessary ${ }^{24)}$. Usually, the axial and radiating pain can be controlled by conservative treatment, such as medication, physiotherapy, restriction of physical activity, use of brace, and selective nerve root blocks ${ }^{7,25}$. However, in patients experiencing uncontrollable pain even with supportive care, the development of neurological or functional disability, progressive slip, and segmental instability are possible indications of surgery ${ }^{7,17,25)}$. The optimal surgical option in isthmic listhesis is still under debate, but one of the most common treatments is decompression and fusion of the lytic segments ${ }^{7,16)}$.

Various studies have reported that the surgical treatment of isthmic spondylolisthesis significantly improves functional outcomes ${ }^{1,716)}$. Through many years of follow-up on surgically treated spondylolytic patients, numerous studies described the surgical results and degeneration of adjacent segments to fused vertebrae $^{2,23,29)}$. Age, genetic factors, high body mass index, multi-level fixation, insufficient lumbar lordosis, osteoporosis, and preoperative disc degeneration of the adjacent segment are known contributors of adjacent segment degeneration after spinal fusion surgery, ${ }^{8,11,26)}$.

The data on surgical treatment and prognosis in patients with MLS and listhesis is very scarce ${ }^{4,14,21,30)}$. Currently there is no report on the natural course of spondylolysis adjacent to the surgically treated isthmic listhesis. Zhang et al. ${ }^{30)}$ and Liu et $\mathrm{al}^{14}{ }^{14)}$ expressed that no variance is needed in the treatment principle between multiple and single isthmic spondylolisthesis. Ravichandran ${ }^{21)}$ described the surgical outcome of fusion in 4 patients with multi-level spondylolysis. Two patients underwent fusion for all the spondylolysis-affected levels, while the other two underwent segmental fusion that did not include all the lysis levels. The group that underwent treatment for all the levels had better results, and thus the authors concluded that radical fusion including all the lytic levels is recommended. Moreover, they mentioned that instability of the non-operated segment could have caused worse outcomes.
Our institute focused on factors that can be managed by the surgeon in order to improve the outcomes. As mentioned above, most of the lytic lesions are somewhat stable and asymptomatic, and the progression of listhesis slows down with time $^{3,24)}$. Therefore, we supposed that under strict indications based on the status of untreated spondylolysis, such as disc and facet joint degeneration, dynamic instability, or lumbopelvic alignment, lessening the fusion level could be a way of reducing perioperative complications or adjacent segment degeneration, eventually leading to better clinical outcomes.

We matched the radiological factors that could affect the degeneration of the neighboring segment after fusion surgery in the MLS and SLS groups. Thus, we compared the prognosis of the untreated non-fused lytic segment with that of the non-operated non-lytic segment. Some authors stated that greater pelvic incidence and sacral slope could aggravate the development of lytic listhesis ${ }^{4}$. Few studies showed that lumbar lordosis is higher in patients with pars defect ${ }^{12}$, and the sacral table angle is related to the incidence of spondylolysis ${ }^{28)}$. The result shown in Table 1 implies that there was no significant difference in the preoperative degeneration of the adjacent segment in either group.

In our cases, we evaluated the anterior and angular instability described by Niggemann et al. ${ }^{15)}$ as the primary outcomes. There was a higher increase of angle motion in the MLS group, and greater slippage in the SLS group. We believe that factors such as bone marrow density and body mass index, which were not evaluated in our study, could have affected the results. Despite the disparity in increment of angular displacement and translation, there was no statistical significance in either group. This shows that instability at the adjacent lysis level is not increased as generally expected with respect to the nonlytic segment during the 2 years of postoperative follow-up. Clinically, the MLS group had higher preoperative NRS scores than the SLS group, but its clinical correlation with severity cannot be assessed based on our data. Lower back pain and leg pain improved in both groups after surgery, but no statistical difference in the decrease of NRS score was noticed at the last follow-up in both groups.

There was one revision case of a 55 -year-old female patient in the MLS group. She had L4 and L5 lysis, and underwent selective surgery on $\mathrm{L} 4 / 5$ only, but needed additional surgery extending the fusion level to S1. Preoperatively, she complained of radiating pain in both legs corresponding to the L4 dermatome. There were no symptoms or instability related to the adjoining untreated lysis preoperatively. Moreover, the MRI showed definite foraminal stenosis only at the L $4 / 5$ level with no significant stenosis at the $\mathrm{L} 5 / \mathrm{S} 1$ level. Postoperatively, she complained of bilateral radiating pain corresponding to a dermatome level different from the preoperative one, presumably caused by the adjacent untreated spondylolysis level. Lumbar dynamogram displayed about $6^{\circ}$ increase of segmental motion immediately after operation as compared with that before the 
surgery, consequently leading to a rise of angular displacement from $9^{\circ}$ to $15^{\circ}$ indicating hypermobility.

This complication in the case indicates that despite no significant difference in the increase in instability from postsurgery to the final follow-up in the MLS and SLS groups, the surgical procedure itself can cause hypermobility, making the adjacent segment vulnerable for additional operation. Thus, considering about $2.4^{\circ}$ escalation of angular motion from before the surgery to after the surgery in the MLS group, selective fusion should be considered in patients who are estimated to have less than $10^{\circ}$ of angular displacement postoperatively. This restriction may reduce the chances of additional surgery caused by instability.

There were several limitations in our study. The study was performed retrospectively, and the sample size was small, especially in patients with triple level lysis. The follow-up period was only about 2 years, which may not be sufficient to observe the long-term outcomes in both groups. In addition, differentiating the symptomatic level from asymptomatic level can be very vague and difficult in actual clinical practice. Moreover, using only the NRS score and dynamogram radiographs as the primary outcomes provided limited information for evaluating the intrinsic effect on the adjacent disc. The use of different types of cages and screws may have affected the postoperative radiological outcome. Other factors that were not evaluated in our study can have contributed to adjacent segment degeneration. Further studies with longer follow-up data and more variables will give us clearer details regarding the fate of the surgically treated MLS patients.

\section{CONCLUSION}

With strict narrow indications, selective fusion in patients with multiple spondylolysis could be a viable option without increasing the risk of adjacent segment degeneration. Additionally, a thorough preoperative evaluation is needed to prevent early surgical failure.

\section{CONFLICTS OF INTEREST}

No potential conflict of interest relevant to this article was reported.

\section{REFERENCES}

1. Alhammoud A, Schroeder G, Aldahamsheh O, Alkhalili K, Lendner M, Moghamis IS, et al.: Functional and radiological outcomes of combined anterior-posterior approach versus posterior alone in management of isthmic spondylolisthesis. A systematic review and meta-analysis. Int J Spine Surg 13:230-238, 2019

2. Bae JS, Lee SH, Kim JS, Jung B, Choi G: Adjacent segment degeneration after lumbar interbody fusion with percutaneous pedicle screw fixation for adult low-grade isthmic spondylolisthesis: minimum 3 years of follow-up. Neurosurgery 67:16001607; discussion 1607-1608, 2010

3. Beutler WJ, Fredrickson BE, Murtland A, Sweeney CA, Grant WD, Baker D: The natural history of spondylolysis and spondylolisthesis: 45-year follow-up evaluation. Spine (Phila Pa 1976) 28:1027-1035; discussion 1035, 2003

4. Darnis A, Launay O, Perrin G, Barrey C: Surgical management of multilevel lumbar spondylolysis: A case report and review of the literature. Orthop Traumatol Surg Res 100:347-351, 2014

5. Dubousset J: Treatment of spondylolysis and spondylolisthesis in children and adolescents. Clin Orthop Relat Res:77-85, 1997

6. Dupuis PR, Yong-Hing K, Cassidy JD, Kirkaldy-Willis WH: Radiologic diagnosis of degenerative lumbar spinal instability. Spine (Phila Pa 1976) 10:262-276, 1985

7. Ganju A: Isthmic spondylolisthesis. Neurosurg Focus 13:E1, 2002

8. Hashimoto K, Aizawa T, Kanno H, Itoi E: Adjacent segment degeneration after fusion spinal surgery-a systematic review. Int Orthop 43:987-993, 2019

9. Hong JY, Suh SW, Modi HN, Hur CY, Song HR, Park JH: Reliability analysis for radiographic measures of lumbar lordosis in adult scoliosis: A case-control study comparing 6 methods. Eur Spine J 19:1551-1557, 2010

10. Inoue $\mathrm{H}$, Ohmori K, Miyasaka K: Radiographic classification of L5 isthmic spondylolisthesis as adolescent or adult vertebral slip. Spine (Phila Pa 1976) 27:831-838, 2002

11. Kim HJ, Kang KT, Chun HJ, Lee CK, Chang BS, Yeom JS: The influence of intrinsic disc degeneration of the adjacent segments on its stress distribution after one-level lumbar fusion. Eur Spine J 24:827-837, 2015

12. Labelle H, Roussouly P, Berthonnaud E, Transfeldt E, O'Brien M, Chopin D, et al.: Spondylolisthesis, pelvic incidence, and spinopelvic balance: A correlation study. Spine (Phila Pa 1976) 29:2049-2054, 2004

13. Legaye J, Duval-Beaupère G, Hecquet J, Marty C: Pelvic incidence: A fundamental pelvic parameter for three-dimensional regulation of spinal sagittal curves. Eur Spine J 7:99-103, 1998

14. Liu X, Wang L, Yuan S, Tian Y, Zheng Y, Li J: Multiple-level lumbar spondylolysis and spondylolisthesis. J Neurosurg Spine 22:283-287, 2015

15. Niggemann P, Kuchta J, Beyer HK, Grosskurth D, Schulze T, Delank KS: Spondylolysis and spondylolisthesis: Prevalence of different forms of instability and clinical implications. Spine (Phila Pa 1976) 36:E1463-E1468, 2011

16. Noorian S, Sorensen K, Cho W: A systematic review of clinical outcomes in surgical treatment of adult isthmic spondylolisthesis. Spine J 18:1441-1454, 2018

17. Omidi-Kashani F, Ebrahimzadeh MH, Salari S: Lumbar spondylolysis and spondylolytic spondylolisthesis: Who should be have surgery? An algorithmic approach. Asian Spine J 8:856-863, 2014

18. Osterman K, Schlenzka D, Poussa M, Seitsalo S, Virta L: Isthmic spondylolisthesis in symptomatic and asymptomatic subjects, epidemiology, and natural history with special reference to disk abnormality and mode of treatment. Clin Orthop Relat Res:6570, 1993

19. Pfirrmann CW, Metzdorf A, Zanetti M, Hodler J, Boos N: 
Magnetic resonance classification of lumbar intervertebral disc degeneration. Spine (Phila Pa 1976) 26:1873-1878, 2001

20. Posner I, White AA, 3rd, Edwards WT, Hayes WC: A biomechanical analysis of the clinical stability of the lumbar and lumbosacral spine. Spine (Phila Pa 1976) 7:374-389, 1982

21. Ravichandran G: Multiple lumbar spondylolyses. Spine (Phila Pa 1976) 5:552-557, 1980

22. Sakai T, Sairyo K, Takao S, Nishitani H, Yasui N: Incidence of lumbar spondylolysis in the general population in Japan based on multidetector computed tomography scans from two thousand subjects. Spine (Phila Pa 1976) 34:2346-2350, 2009

23. Sakaura H, Yamashita T, Miwa T, Ohzono K, Ohwada T: Symptomatic adjacent segment pathology after posterior lumbar interbody fusion for adult low-grade isthmic spondylolisthesis. Global Spine J 3:219-224, 2013

24. Sedney CL, McConda DB, Daffner SD: Natural history of spondylolysis and spondylolisthesis. Semin Spine Surg 26:214-218, 2014

25. Syrmou E, Tsitsopoulos PP, Marinopoulos D, Tsonidis C, Anagnostopoulos I, Tsitsopoulos PD: Spondylolysis: A review and reappraisal. Hippokratia 14:17-21, 2010

26. Wang H, Ma L, Yang D, Wang T, Liu S, Yang S, et al.: Incidence and risk factors of adjacent segment disease following posterior decompression and instrumented fusion for degenerative lumbar disorders. Medicine (Baltimore) 96:e6032, 2017

27. Weishaupt D, Zanetti M, Boos N, Hodler J: MR imaging and CT in osteoarthritis of the lumbar facet joints. Skeletal Radiol 28:215-219, 1999

28. Whitesides TE, Jr., Horton WC, Hutton WC, Hodges L: Spondylolytic spondylolisthesis: A study of pelvic and lumbosacral parameters of possible etiologic effect in two genetically and geographically distinct groups with high occurrence. Spine (Phila Pa 1976) 30:S12-S21, 2005

29. Yu CH, Lee JE, Yang JJ, Chang BS, Lee CK: Adjacent segment degeneration after single-level PLIF: Comparison between spondylolytic spondylolisthesis, degenerative spondylolisthesis and spinal stenosis. Asian Spine J 5:82-90, 2011

30. Zhang S, Ye C, Lai Q, Yu X, Liu X, Nie T, et al.: Double-level lumbar spondylolysis and spondylolisthesis: A retrospective study. J Orthop Surg Res 13:55, 2018 